\title{
Stapled Sequences and Stapling Coverings of Natural Numbers
}

\author{
Irene Gassko* \\ irina@cs.bu.edu \\ Boston University \\ Computer Science Department
}

AMS Subject Classification: 11B50(primary),

11A07, 11Y16, 11Y55, 68R05, 11B75 (secondary).

Submitted: March 30, 1996; Accepted: October 30, 1996

\begin{abstract}
A stapled sequence is a set of consecutive positive integers such that no one of them is relatively prime with all of the others. The problem of existence and construction of stapled sequences of length $N$ was extensively studied for over 60 years by Pillai, Evans, Brauer, Harborth, Erdös and others.

Sivasankaranarayana, Szekeres and Pillai proved that no stapled sequences exist for any $N<17$. We give a new simple proof of this fact.

There exist several proofs that stapled sequences exist for any $N \geq 17$. We show that existence of stapled sequences is equivalent to existence of stapling coverings of a sequence of $N$ consecutive natural numbers by prime arithmetic progressions such that each progression has at least two common elements with the sequence and discuss properties of stapling coverings. We introduce the concept of efficiency of stapling coverings and develop algorithms that produce efficient stapling coverings. Using the result by Erdös, we show that the greatest prime number used in stapling coverings of length $N$ can be made $o(N)$.
\end{abstract}

*Partially supported by NSF grant CCR-9204284 
THE ELECTRONiC JOURNAL of COMBinAtorics 3 (1996), \#R33

\section{Introduction}

Consider the following problem: for a given $N$, does there exist a sequence $S_{N}$ of $N$ successive natural numbers such that no element is relatively prime with all the others? (We call such sequences stapled.) This problem was originally suggested by Szekeres [4] and by Pillai [13]. It was extensively studied for over half a century by Erdös, Pillai, Evans, Brauer, Harborth and others. Sivasankaranarayana, Szekeres and Pillai proved that no stapled sequences exist for any $N<17$ [5]. A simpler proof of this fact is presented in this paper. Pillai [13], Brauer [1], Harborth [10,11] and Evans[2] proved that for any $N \geq$ 17 there exist stapled sequences of length $N$, i.e. sequences of consecutive natural numbers, where each element has a common divisor $1<d \leq N$ with the product of all the other elements of the sequence. As shown below, this problem is equivalent to the problem of covering finite sequences of natural numbers by arithmetic progressions with prime differences. The concept of efficiency of such coverings is introduced in this paper and constructions producing efficient stapling coverings are presented.

While Evans' solution [2] is considered the most elegant proof of the existence of stapling coverings for $N>16$, Brauer's solution [1] is seemingly the most efficient one suggested before this paper. Below we describe algorithms that produce significantly more efficient coverings than those by Brauer. It is also shown that the greatest prime number used in a in stapling covering can be made smaller than $\delta N$, for any $\delta>0$, if $N$ is sufficiently large.

\section{Definitions}

Definition 2.1. A sequence of successive natural numbers (SSN) $S_{N}$ of length $N$ is called a stapled sequence if for any $s \in S_{N} \exists s^{\prime} \in S_{N}, s^{\prime} \neq s$, such that the greatest common divisor $\left(s, s^{\prime}\right)>1$.

Definition 2.2. An arithmetic progression $A_{p}^{a_{p}}=\left\{a_{p}+k p \mid k \in \mathbb{Z}\right\}\left(a_{p} \in\right.$ $\mathbb{Z}_{p}$ ) is called a prime congruence if $p$ is prime. (The upper index $a_{p}$ will be omitted whenever it is not essential).

Denote by $p_{i}$ the i-th prime number.

Definition 2.3. Consider a set of congruences $W_{I}=\left\{A_{p_{i}}\right\}(I=\{i\} \subseteq \mathbb{N})$. The set $T=T\left(S_{N}, W_{I}\right)=\left\{V_{i} \mid i \in I\right\}$ where $V_{i}=S_{N} \bigcap A_{p_{i}}$ is called a tiling of $S_{N}$ by $W_{I}$. If all $p_{i}$ are distinct primes, $T$ is a prime tiling. 


$$
\text { Obviously, } U=U\left(S_{N}, W_{I}\right)=\bigcup_{i \in I}\left(S_{N} \bigcap A_{p_{i}}\right) \subseteq S_{N} \text {. }
$$

Definition 2.4. A tiling $T=T\left(S_{N}, W_{I}\right)$ is complete if $U=S_{N}$. A complete tiling is called also a covering of $S_{N}$ by $W_{I}$.

Definition 2.5. If in a prime covering $T\left(S_{N}, W_{I}\right)$

$$
\left|S_{N} \bigcap A_{p_{i}}\right| \geq 2, \text { for any } i \in I
$$

$T$ is called a stapling covering of $S_{N}$ by $W_{I}$.

If $\left|S_{N} \bigcap A_{p_{i}}\right| \geq n$, for any $i \in I, n \geq 2$, $T$ is called an n-stapling covering of $S_{N}$ by $W_{I}$.

Definition 2.6. Consider $S_{N}=\left(s_{1}, s_{2}, \ldots, s_{N}\right)$ and a prime tiling $T\left(S_{N}, W_{I}\right)$. If $s_{r_{i}} \in A_{p_{i}}$ the number $r_{i}$ is called indicator of $A_{p_{i}}$ in $S_{N}$. If $h_{i}=h\left(p_{i}\right)$ is the least number such that $s_{h_{i}} \in A_{p_{i}}, h_{i}$ is the first indicator of $A_{p_{i}}$ in $S_{N}$. If $s_{r} \in A_{p}$ we say that $A_{p}$ (or, simply, $p$ ) covers $s_{r}$.

Obviously, a tiling of $S_{N}$ is uniquely determined by the set of its first indicators $\left\{h_{i} \mid i \in I\right\}$.

Definition 2.7. Two $S S N^{\prime} s S_{N}$ and $S_{N}^{\prime}$ are equivalent with respect to $W_{I}$ $\left(S_{N} \sim S_{N}^{\prime}\left(\operatorname{resp} W_{I}\right)\right)$, if for any $i \in I, h_{i}=h_{i}^{\prime}$, where $s_{h_{i}} \in S_{N}, s_{h_{i}^{\prime}} \in S_{N}^{\prime}$.

\section{Example}

The shortest, and, seemingly, the first known example of a stapled sequence is the sequence of length $N=17$ which starts with $s_{1}=2184$ and ends with $s_{17}=2200$ (we denote it by $S=[2184,2200]$ ). Let us use this example to illustrate the notation in Defs. 2.1 to 2.7 .

The stapling covering of this sequence is given by a set of congruences $W_{I}=\left\{A_{p_{i}}^{0}\right\}$, where $I=\{1,2,3,4,5,6\}, p_{1}=2, p_{2}=3, p_{3}=5, p_{4}=7$, $p_{5}=11, p_{6}=13$. The first indicators are as follows: $h_{1}=h(2)=1$ (which means that $s_{1}=2184$ is divisible by $\left.2: s_{1}=2184 \in A_{2}^{0}\right) ; h_{2}=h(3)=1$ $\left(s_{1} \in A_{3}^{0}\right) ; h_{3}=h(5)=2\left(s_{2}=2185 \in A_{5}^{0}\right) ; h_{4}=h(7)=1\left(s_{1} \in A_{7}^{0}\right)$; $h_{5}=h(11)=6\left(s_{6} \in A_{11}^{0}\right) ; h_{6}=h(13)=1\left(s_{1} \in A_{13}^{0}\right)$.

This stapled sequence is equivalent to the sequence $[2184+30030 k, 2200+$ $30030 k], k \in \mathbb{Z}$, with respect to the same set of congruences, where 30030 is the least common multiple of $2,3,5,7,11,13$.

The same set of first indicators provides stapling covering for any SSN of length $N$, but, of course, with shifted prime congruences. In particular, 
stapling covering for the sequence $[1,17]$ is given by $A_{2}^{1}, A_{3}^{1}, A_{5}^{2}, A_{7}^{1}, A_{11}^{6}, A_{13}^{1}$ as shown below

\begin{tabular}{|l|rrrrrrrrrrrrrrrrr|}
\hline$s_{i}$ & 1 & 2 & 3 & 4 & 5 & 6 & 7 & 8 & 9 & 10 & 11 & 12 & 13 & 14 & 15 & 16 & 17 \\
\hline$p_{1}$ & 2 & & 2 & & 2 & & 2 & & 2 & & 2 & & 2 & & 2 & & 2 \\
$p_{2}$ & 3 & & & 3 & & & 3 & & & 3 & & & 3 & & & 3 & \\
$p_{3}$ & & 5 & & & & & 5 & & & & & 5 & & & & & 5 \\
$p_{4}$ & 7 & & & & & & & 7 & & & & & & & 7 & & \\
$p_{5}$ & & & & & & 11 & & & & & & & & & & & 11 \\
$p_{6}$ & 13 & & & & & & & & & & & & & 13 & & & \\
\hline
\end{tabular}

\section{$3 \quad$ Properties of Stapled Sequences}

Denote by $W_{I}^{0}$ a set of prime congruences $A_{p_{i}}^{0}$, such that $A_{p_{i}}^{0}=k p_{i}, k \in \mathbb{Z}$, $i \in I$, i.e. such that $a_{p_{i}}=0, i \in I$. Obviously, if $T\left(S_{N}^{0}, W_{I}^{0}\right)$ is a stapling covering, then $S_{N}^{0}$ is a stapled sequence.

Lemma 3.1. If $S_{N}^{0}$ is a stapled $S S N$ and $T\left(S_{N}^{0}, W_{I}^{0}\right)$ is the corresponding stapling covering, there exists stapling covering $T\left([1, N], W_{I}\right)$ such that $s_{h_{I}}=$ $h_{i}=h_{i}^{0}$ for any $i \in I$.

The example given at the end of the Sec. 2 illustrates this lemma. The position of the first term divisible by $p_{i}$ in a stapled sequence is equal to the first indicator (i.e. to the "shift" $a_{p_{i}}$ ) of $A_{p_{i}}$ in the stapling covering for $[1, N]$.

Lemma 3.2. If $T\left(S_{N}, W_{I}\right)$ is a stapling covering, then there exists a stapled $S S N S_{N}^{0}$ of length $N$.

Lemmata 3.1 and 3.2 show that the existence of a stapling covering of the sequence $[1, N]=(1,2, \ldots, N)$ is the necessary and sufficient condition for the existence of a stapled sequence of length $N$.

If $T\left([1, N], W_{I}\right)$ is a stapling covering with a set of first indicators $\left\{h_{i}\right\}$, $i \in I$, then a stapled sequence of length $N$ is $S_{N}=(M+1, \ldots, M+N)$, where $\mathrm{M}$ satisfies equations:

$$
M+h_{i} \equiv 0 \quad \bmod p_{i}
$$


Lemma 3.3. $S_{N} \sim S_{N}^{\prime}\left(\right.$ resp $\left.W_{I}\right)$ iff $s_{k}^{\prime} \equiv s_{k}\left(\bmod \prod_{i \in I} p_{i}\right)$, for all $k \in$ $[1, N]=(1,2, \ldots, N), s_{k} \in S_{N}, s_{k}^{\prime} \in S_{N}^{\prime}$.

Proofs of Lemmata 3.1, 3.2 and 3.3 are given in Appendix A.

Lemmata 3.2 and 3.3 show, in particular, that if for a given $N$ there exists one stapled sequence, then there exist infinitely many of them.

Note, that if there exists a stapling covering $T\left(S_{N}, W_{I}\right)$, then there exists its "mirror image", i.e. stapling covering $T^{\prime}\left(S_{N}, W_{I}^{\prime}\right)$ such that if $s_{r} \in A_{p}$ in $T$ then $s_{N-r+1} \in A_{p}$ in $T^{\prime}$.

Lemma 3.4. A covering $T\left(S_{N}, W_{I}\right)$ and its "mirror image" $T^{\prime}\left(S_{N}, W_{I}^{\prime}\right)$ are always different.

Proof. Let us show that a covering cannot be symmetric, i.e. cannot be identical with its mirror image. Indeed, if $N$ is even then $s_{\frac{1}{2} N}$ and $s_{\frac{1}{2} N+1}$ cannot be covered by the same primes thus breaking symmetry. If $N$ is odd and the stapling covering is symmetric, then $s_{\frac{1}{2}(N+1)}$ must be covered by all $A_{p} \in W_{I}$, where $\mathrm{p}$ is odd. Indeed, if both $s_{r}$ and $s_{N-r+1}$ are covered by an odd prime $p$, then $s_{N-r+1}-s_{r}=N+1-2 r=2 k p, k \in \mathbb{Z}$. Hence, $s_{\frac{1}{2}(N+1)}-s_{r}=\frac{1}{2}(N+1-2 r)=k p$, and $s_{\frac{1}{2}(N+1)}$ is covered by $p$. Then $s_{\frac{1}{2}(N-1)}$ and $s_{\frac{1}{2}(N-3)}$ are not covered by any odd $p$. But only one of these numbers can be covered by 2 . Thus, symmetric coverings are impossible, which proves the lemma.

Corollary 3.5. The number of different stapling coverings of length $N$ is always even.

Proof. Follows immediatedly from Lemma 3.4.

Lemma 3.6. If $S_{2 N}$ is a stapled $S S N$, then there exist $S_{2 N+1}$ and $S_{2 N-1}$ which are also stapled.

Proof. - If there exists stapled $S_{2 N}$, it means that there exists a stapling covering $T\left([1,2 N], W_{I}\right)$. If $h(2)=1$, then $2 N+1 \in A_{2}$, and the lemma is proved. If $h(2)=2$, then consider $W_{I}^{\prime}=\left\{A_{p_{i}}^{\prime}\right\}$ where for each $A_{p_{i}}^{\prime} h_{i}^{\prime}=$ $h_{i}+1\left(\bmod p_{i}\right)$. These progressions form a stapling covering of the sequence $(2, \ldots, 2 N+1)$. Since, obviously, $1 \in A_{2}^{\prime},\left([1,2 N+1], W_{I}\right)$ is a stapling covering, and a stapled $S_{2 N+1}$ exists.

- Without loss of generality assume that in $\left([1,2 N], W_{I}\right) h(2)=1$. (If $h(2)=2$, consider the "mirror image" of the sequence). Then $2 N$ is covered 
by $A_{p}$, where $p$ is an odd prime. If $p<N,\left|[1,2 N-1] \bigcap A_{p}\right| \geq 2$. If $p \geq N$, the only $r \in A_{p}, r \neq 2 N$ is odd and, hence, $r \in A_{2}$ and $A_{p}$ can be removed from $W_{I}$. Thus, the number $2 N$ can be deleted without violating the stapling condition, and stapled $S_{2 N-1}$ exists.

Lemma 3.7. If $S_{2 N-1}$ is a stapled $S S N$ and $2 N-1$ is prime, there exists $S_{2 N}$ which is also stapled.

Proof. Consider a stapling covering $T\left([1,2 N-1], W_{I}\right)$. Then a stapling covering of $[1,2 N]$ is given by $W_{I}^{\prime}$, where $W_{I}^{\prime}=W_{I} \bigcup\left\{A_{2 N-1}\right\}, h(2 N-1)=1$. Thus, a stapled $S_{2 N}$ exists.

Theorem 3.8. There exist no stapled SSN of lengths $N \leq 16$.

In other words, any SSN of length $N \leq 16$ includes a member relatively prime with all other members.

Proof. It follows from Lemmata 3.6 and 3.7 that it is sufficient to prove the theorem for $N=15$ and $N=9$.

For $N=15$, note that if there exists a stapling covering of $[1,15]$ where $a_{2}=2, h(2)=2$, then there exists a stapling covering of $[2,16]$ with $a_{2}=2$, $h(2)=1$. Thus it is sufficient to show that no such stapling covering of $[2,16]$ exists.

Suppose first that $a_{3}=3$. Then each of $A_{5}, A_{7}, A_{11}$ can cover only one of the numbers $5,7,11,13$, and $A_{13}$ can cover none. Thus, $a_{3}=5$ or $a_{3}=7$. Because of "mirror image" symmetry, it is enough to consider $a_{3}=5$. Now, $3,7,9,13,15$ remain to be covered, and $A_{5}$ must cover two of them. Hence $a_{5}=3$. Then neither 7 nor 9 can be covered by $A_{11}$ or $A_{13}$, and both of them cannot be covered simultaneously by $A_{7}$, thereby making stapling covering impossible. Thus no stapling covering of length 15 exists.

For $N=9$ it is readily seen that $A_{2}$ can cover either four or five numbers. If $A_{2}$ covers four numbers, then $A_{3}, A_{5}$ and $A_{7}$ can cover not more than two numbers, one number, and one number, respectively, out of five remaining numbers, thus, leaving one number not covered. If $A_{2}$ covers five numbers, then the only way to cover two numbers with $A_{3}$ is to choose $h(3)=2$. However, since $A_{7}$ cannot cover 4 or 6 , again one number is left not covered. Thus, stapling coverings do not exist for $N=9$, which completes the proof. 
For $N=17$ there exist only two different stapling coverings which are mirror images of each other. One is given by first indicators $(1,2,1,3,1,4)$ (i.e., $\left.h_{1}=h(2)=1, h_{2}=h(3)=2, \ldots, h_{6}=h(13)=4\right)$. The other is given by $(1,1,2,1,6,1)$ (Cf. example in Sec. 2). It follows then, by Lemmata 3.6 and 3.7, that stapling coverings exist for $17 \leq N \leq 21$. It is remarkable that, as computer calculations show, it is possible to extend the stapling covering given by $(1,2,1,3,1,4)$ to the right in order to construct stapling coverings up to $N \leq 4 \cdot 10^{7}$, and, most probably, for all larger $N$. More exactly, the procedure is the following. We start with stapling covering for $S_{17} \in[1,17]$ given by the set of prime congruences with first indicators $(1,2,1,3,1,4)$. At each step we go from $S_{N}=[1, N]$ to $S_{N+1}=[1, N+1]$ and check whether the last number $N+1$ is covered by at least one of the prime congruences used in the stapling covering of $S_{N}$. If this is not so, we use the smallest unused prime number $p<N+1$ to cover $N+1$ and add the prime congruence $A_{p}$ to the set $W_{I}$. This approach, however, does not work if one starts with the set of congruences given by first indicators $(1,1,2,1,6,1)$ : this set cannot be extended for $N=25$.

In fact, as shown below, stapling coverings exist for all $N \geq 17$.

\section{Efficient Stapling Coverings}

An interesting characteristic of stapling covering is the ratio of the number $|I|$ of primes used for the covering to the total number $\pi(N)$ of primes not exceeding $N$.

Definition 4.1. The expense $\varepsilon(T)$ of a stapling covering $T\left(S_{N}, W_{I}\right)$ is the ratio $\varepsilon(T)=\frac{|I|}{\pi(N)}$.

Stapling coverings with expense substantially smaller than 1 are called efficient.

Another related characteristic is cutoff.

Definition 4.2. The cutoff $u(T)$ of a stapling covering $T\left(S_{N}, W_{I}\right)$ is the ratio of the greatest prime $p_{i}, i \in I$ to $N$.

It is easy to see that the coverings with the small cutoff are efficient. It is an interesting open problem though to show that efficient stapling coverings can always be transformed into coverings of small cutoff. 
It is worth to note that the simple approach described in the Sec. 3 yields rather efficient stapling coverings for large $N$. The expense $\varepsilon(T)$ decreases with $N$ from $\frac{\pi(N)-1}{\pi(N)}=\frac{6}{7}$ for $N=17$ to approximately 0.62 for $N=4 \cdot 10^{7}$. However, if $N$ is sufficiently large, stapling coverings with substantialy smaller expense and cutoff become possible. The construction given by Brauer [1] uses a sequence of integers $S_{N}$ which is symmetric with respect to zero and achieves $u(T)=1 / 2$. The use of symmetry, however, may be inconvenient in some related problems. Therefore, we provide a construction that yields $u(T)=1 / 2$ without use of symmetry.

Lemma 4.1. Consider the set $Q=\left\{2^{s} 3^{t} \mid s, t \in \mathbb{N}, 2^{s} 3^{t} \leq N\right\}$.

Then $|Q| \leq \frac{1}{2} \log _{2} N\left(\log _{3} N-1\right)$ for any $N \geq 9$.

Proof of Lemma 4.1 is given in Appendix B.

Theorem 4.2. There exists a stapling covering $T=T\left(S_{N}, W_{I}\right)$ for all $N$ such that

$$
\pi\left(\left\lfloor\frac{N}{2}\right\rfloor\right)-\pi\left(\left\lfloor\frac{N}{4}\right\rfloor\right) \geq \log _{2} N \cdot \log _{3} N
$$

The covering has the property that $p_{i} \leq \frac{N}{2}$ for all $i \in I$ and $\lim _{N \rightarrow \infty} \varepsilon(T) \leq \frac{3}{8}$.

Proof. Let $p_{i}$ be the $i$-th prime number: $p_{1}=2, p_{2}=3$, etc. Consider the following procedure of covering the sequence $S_{N}=(1,2, \ldots, N)$.

1. $h_{i}=p_{i}$ for all $p_{i} \leq \frac{N}{4}, p_{i} \neq 2,3$.

2. $h_{1}=h(2)=1$.

3. Denote:

$$
\begin{aligned}
& P_{1}=\left\{p_{i} \mid 2 p_{i} \equiv 1(\bmod 3), \frac{N}{4}<p_{i} \leq \frac{N}{2}\right\} \\
& P_{2}=\left\{p_{i} \mid 2 p_{i} \equiv 2(\bmod 3), \frac{N}{4}<p_{i} \leq \frac{N}{2}\right\} \\
& D_{1}=\left\{2^{2 k} \mid k \in \mathbb{N}, 2 k \leq \log _{2} N\right\} \\
& D_{2}=\left\{2^{2 k-1} \mid k \in \mathbb{N}, 2 k-1 \leq \log _{2} N\right\}
\end{aligned}
$$

Choose

$$
h_{2}=h(3)= \begin{cases}1, & \text { if }\left|P_{1}\right|+\left|D_{1}\right| \geq\left|P_{2}\right|+\left|D_{2}\right| \\ 2, & \text { otherwise }\end{cases}
$$


4. $h_{i}=p_{i}$, if $h(3)=1$ and $p_{i} \in P_{2}$, or if $h(3)=2$ and $p_{i} \in P_{1}$.

5. Denote: $Q=\left\{2^{s} 3^{t} \mid s, t \in \mathbb{N}, 2^{s} 3^{t} \leq N\right\}$

If $h(3)=1$, use members of $P_{1}$ to cover as many as possible members of $D_{2} \cup Q$.

If $h(3)=2$, use members of $P_{2}$ to cover as many as possible members of $D_{1} \cup Q$.

(It will be shown below that under condition (4.1) it is possible to cover all members of $D_{2} \bigcup Q$ or $D_{1} \bigcup Q$, respectively).

Note that since $p \leq \frac{N}{2}$ if $p \in P_{1}$ or $p \in P_{2},\left|S_{N} \bigcap A_{p}\right| \geq 2$ for any choice of $h(p)$. As a result, we obtain a prime tiling $T\left(S_{N}, W_{I}\right)$, which satisfies the stapling condition (2.1). In this tiling, $A_{2}$ covers all odd numbers, $A_{3}$ covers all even numbers belonging to $2 P_{1} \cup D_{1}$, if $h(3)=1$, or to $2 P_{2} \bigcup D_{2}$, if $h(3)=2$. All other even numbers, except members of $D_{2} \cup Q$, if $h(3)=1$, or $D_{1} \cup Q$, if $h(3)=2$, are covered by "unmoved" prime numbers for which $h_{i}=p_{i}$. It remains to show that the set $P_{1}$ (respectively, $P_{2}$ ) is large enough to cover all members of $D_{2} \cup Q$ (respectively, $D_{1} \cup Q$ ).

Without loss of generality, assume that $h(3)=1$ and, thus $\left|P_{1}\right|+\left|D_{1}\right| \geq$ $\left|P_{2}\right|+\left|D_{2}\right|$. Then $\left|P_{1}\right|-\left|D_{2}\right| \geq\left|P_{2}\right|-\left|D_{1}\right|$. Since $\left|P_{1}\right|+\left|P_{2}\right|=$ $\pi\left(\left\lfloor\frac{N}{2}\right\rfloor\right)-\pi\left(\left\lfloor\frac{N}{4}\right\rfloor\right)$, and $\left|D_{1}\right|+\left|D_{2}\right|=\left\lfloor\log _{2} N\right\rfloor$, it follows that

$$
\left|P_{1}\right|-\left|D_{2}\right| \geq \frac{1}{2}\left[\pi\left(\left\lfloor\frac{N}{2}\right\rfloor\right)-\pi\left(\left\lfloor\frac{N}{4}\right\rfloor\right)-\log _{2} N\right]
$$

By lemma 4.1,

$$
|Q| \leq \frac{\log _{2} N\left(\log _{3} N-1\right)}{2}
$$

Now, taking into account (4.1), (4.2) and (4.3), we obtain:

$$
\begin{array}{r}
\left|P_{1}\right| \geq \frac{1}{2}\left[\pi\left(\left\lfloor\frac{N}{2}\right\rfloor\right)-\pi\left(\left\lfloor\frac{N}{4}\right\rfloor\right)-\log _{2} N\right]+\left|D_{2}\right| \\
\geq \frac{1}{2}\left(\log _{2} N \log _{3} N-\log _{2} N\right)+\left|D_{2}\right| \geq|Q|+\left|D_{2}\right|=\left|D_{2} \bigcup Q\right|
\end{array}
$$

Thus, condition (4.1) guarantees that the obtained prime tiling is a stapling covering. Since

$$
\frac{1}{2}\left[\pi\left(\left\lfloor\frac{N}{2}\right\rfloor\right)-\pi\left(\left\lfloor\frac{N}{4}\right\rfloor\right)-\log _{2} N\right] \geq \frac{N}{4 \ln N}
$$


THE ELECTRONiC JOURNAL of COMBinAtorics 3 (1996), \#R33

(cf. [12]), condition (4.1) is fulfilled for sufficiently large $N$. Furthermore, for large $N$ the expense approaches $\frac{3}{8}$. Indeed, using the Prime Number Theorem ([12], p.36), we obtain

$$
\begin{array}{cc}
\varepsilon(T)= & \frac{|I|}{\pi(N)} \leq \frac{1}{\pi(N)}\left[\pi\left(\left\lfloor\frac{N}{4}\right\rfloor\right)+\frac{1}{2}\left(\pi\left(\left\lfloor\frac{N}{2}\right\rfloor\right)-\right.\right. \\
\left.\left.\pi\left(\left\lfloor\frac{N}{4}\right\rfloor\right)+\log _{2} N \log _{3} N\right)\right]=\frac{3}{8}+\frac{\ln 2}{2 \ln N}+O\left(\frac{\ln ^{3} N}{N}\right)
\end{array}
$$

It follows from the Prime Number Theorem that inequality (4.1) is fulfilled for all sufficienly large $N$. Computer test shows that (4.1) is valid for all $N \geq 2098$ and the above algorithm works for all $N \geq 1618$.

Corollary 4.3. Stapled sequences of natural numbers exist for all $N \geq 17$.

Proof. Follows from the results of Sec. 3 and Theorem 4.2.

The construction given in the Theorem 4.2 can be amended by choosing properly indicators for other small prime numbers in order to lower expense and cutoff. However, the same goal can be achieved easier by use of symmetry (somewhat similar to Brauer's approach).

Lemma 4.4. Let

$$
G=\left\{x\left|x= \pm 2^{s} 3^{t} 5^{v},\right| x \mid \leq \frac{N}{2} ; s \in \mathbb{Z} ; t, v \in \mathbb{N} \cup 0 ; x \equiv 2(\bmod 3)\right\}
$$

Then

$$
|G|<\frac{1}{3} \log _{2} \frac{N}{2} \log _{3} \frac{N \sqrt{5}}{2} \log _{5} \frac{5 N}{2}+1
$$

Proof of Lemma 4.4 is given in Appendix C.

Theorem 4.5. There exists a stapling covering $T=T\left(S_{N}, W_{I}\right)$ for all $N$ such that

$$
\pi\left(\left\lfloor\frac{N}{4}\right\rfloor\right)-\pi\left(\left\lfloor\frac{N}{8}\right\rfloor\right) \geq \frac{4}{3} \log _{2} \frac{N}{2} \cdot \log _{3} \frac{N \sqrt{5}}{2} \cdot \log _{5} \frac{5 N}{2}
$$

which has the property that $p_{i} \leq \frac{N}{4}$ for any $i \in I$ and $\lim _{N \rightarrow \infty} \varepsilon(T) \leq \frac{7}{32}$. 
Proof. Let $p$ be a prime number, $p \leq N$. Consider the following procedure of covering the sequence

$$
S_{N}=\left(-\left\lfloor\frac{N-1}{2}\right\rfloor,-\left\lfloor\frac{N-1}{2}\right\rfloor+1, \ldots,-1,0,1, \ldots,\left\lfloor\frac{N}{2}\right\rfloor\right) .
$$

1. Choose: $a_{2}=1 ; a_{3}=1$.

2. Denote:

$$
\begin{aligned}
& P=\left\{p \mid \frac{N}{8}<p \leq \frac{N}{4}\right\} \\
& R_{a_{5}}=\left\{p \mid\{2 p,-2 p\} \subset A_{3}^{1} \cup A_{5}^{a_{5}}, \frac{N}{8}<p \leq \frac{N}{4}\right\}
\end{aligned}
$$

Since for any $p$ either $2 p$ or $-2 p$ is covered by $A_{3}^{1}$, and the other element of the pair $\{2 p,-2 p\}$ belongs to one of the sets $R_{a_{5}}\left(a_{5}=1,2,3,4\right)$, $\bigcup_{a_{5}} R_{a_{5}}=P$, and, therefore, there exists $a_{5}=b$, such that

$$
\left|R_{b}\right| \geq \frac{1}{4}|P|=\frac{1}{4}\left[\pi\left(\left\lfloor\frac{N}{4}\right\rfloor\right)-\pi\left(\left\lfloor\frac{N}{8}\right\rfloor\right)\right]
$$

Choose $a_{5}=b$;

Note that now all primes belonging to $R_{b}$ are free, that is, all their multiples belonging to $S_{N}$ are covered by other prime congruences. Therefore these primes can be shifted to cover other numbers.

3. Let

$$
H=\left\{x\left|x= \pm 2^{s} 3^{t} 5^{v} ; s, t, v \in \mathbb{N} \cup 0 ;\right| x \mid \leq N / 2 ; x \notin A_{2}^{1} \cup A_{3}^{1} \cup A_{5}^{b}\right\}
$$

Members of $H$ remain not covered after 2, 3, and 5 have been shifted. Fortunately, this set is rather small: it is not difficult to show that

$$
|H| \leq|G|-1 \text { for any } N \geq 16
$$

Hence, by lemma 4.4,

$$
|H|<\frac{1}{3} \log _{2} \frac{N}{2} \cdot \log _{3} \frac{N \sqrt{5}}{2} \cdot \log _{5} \frac{5 N}{2}
$$


THE ELECTRONiC JOURNAL of COMBinAtorics 3 (1996), \#R33

Since $\pi(N) \sim \frac{N}{\ln N}$, by the Prime Number Theorem, for sufficiently large $N$,

$$
\left|R_{b}\right| \geq \frac{1}{4}\left[\pi\left(\left\lfloor\frac{N}{4}\right\rfloor\right)-\pi\left(\left\lfloor\frac{N}{8}\right\rfloor\right)\right] \geq \frac{1}{3} \log _{2} \frac{N}{2} \cdot \log _{3} \frac{N \sqrt{5}}{2} \cdot \log _{5} \frac{5 N}{2}>|H|
$$

4. Choose $D \subseteq R_{b}$ such that $|D|=|H|$ and let $f: D \rightarrow H$ be a bijection. Take $a_{p}=q$, where $q=f(p)$, if $p \in D$, and $a_{p}=0$ for all $p \leq \frac{N}{4}$, $p \notin D \cup\{2,3,5\}$.

As a result, we have obtained a stapling covering of the sequence $S_{N}$ (4.9) which uses only prime numbers $p \leq \frac{N}{4}$.

Since

$\lim _{N \rightarrow \infty} \frac{|H|}{\pi(N)}=0, \lim _{N \rightarrow \infty} \frac{\left|R_{b}\right|}{\pi(N)} \geq \frac{1}{32}$, and $\lim _{N \rightarrow \infty} \frac{\pi\left(\frac{N}{4}\right)}{\pi(N)}=\frac{1}{4}$,

it follows that $\lim _{N \rightarrow \infty} \varepsilon(N) \leq \frac{7}{32}$.

As seen from 4.13 this algorithm works for all $N$ such that

$$
\pi\left(\left\lfloor\frac{N}{4}\right\rfloor\right)-\pi\left(\left\lfloor\frac{N}{8}\right\rfloor\right) \geq \frac{4}{3} \log _{2} \frac{N}{2} \cdot \log _{3} \frac{N \sqrt{5}}{2} \cdot \log _{5} \frac{5 N}{2}
$$

The constructions given in Theorems 4.2, 4.5 can generate exponentially large (in $N$ ) number of different stapling coverings.

In the first draft of this paper the author conjectured, that for any $\delta>$ 0 there exist stapling coverings that do not use moduli greater than $\delta N$. According to P. Erdös [7] this is indeed true and follows from his theorem in [6]. We quote the theorem here:

Theorem 4.6. For a certain positive constant $c_{2}$, we can find $c_{2} p_{n} \log p_{n} /\left(\log \log p_{n}\right)^{2}$ consecutive integers so that no one of them is relatively prime to the product $p_{1} p_{2} \cdots p_{n}$, i.e. each of these integers is divisible by at least one of the primes $p_{1}, p_{2}, \cdots, p_{n}$. 
THE ELECTRONiC JOURNAL of COMBinAtorics 3 (1996), \#R33

(Here log stands for the natural logarithm).

Using this fact it can be readily proved that our conjecture holds, i.e. the following theorem is true:

Theorem 4.7. For every $\delta>0$ there exists $N(\delta)$ such that for any $N>$ $N(\delta)$ there is a stapled sequence of length $N$ which has a stapling covering with the largest modulus less than $\delta N$.

Proof. Let $p_{m}$ be the smallest prime such that

$$
\frac{\left(\ln \ln p_{m}\right)^{2}}{c_{2} \ln p_{m}} \leq \frac{\delta}{2}
$$

Denote

$$
\left\lfloor\frac{c_{2} p_{m} \ln p_{m}}{\left(\ln \ln p_{m}\right)^{2}}\right\rfloor=N(\delta)
$$

Let $N>N(\delta)$, and $p_{n}$ be the smallest prime such that

$$
\frac{c_{2} p_{n} \ln p_{n}}{\left(\ln \ln p_{n}\right)^{2}} \geq N
$$

Then, $p_{n}>p_{m}$, and, therefore, $p_{n-1} \geq p_{m}$. Since $\frac{\left(\ln \ln p_{i}\right)^{2}}{c_{2} \ln p_{i}}$ decreases monotonically as $p_{i}$ grows, it follows from (4.15) that

$$
\frac{\left(\ln \ln p_{n-1}\right)^{2}}{c_{2} \ln p_{n-1}} \leq \frac{\delta}{2}
$$

Hence, by (4.17),

$$
p_{n-1} \leq \frac{\delta N}{2}
$$

As well known, $p_{n}<2 p_{n-1}$. Thus, $p_{n}<\delta N$.

It follows from (4.17) and theorem 4.6 that there exists a sequence $S_{N}$ of $N$ consecutive natural numbers such that each of them is divisible by at least one of the primes $p_{1}, p_{2}, \ldots, p_{n}$. Thus, $S_{N}$ is a stapled sequence with the cutoff $u<\delta$. 
This result, however, does not provide an efficient algorithm for constructing such a sequence.

In fact, it is possible, by a slight modification of the proof (namely, choosing $p_{m}$ such that $\left.\frac{\left(\ln \ln p_{m}\right)^{3}}{c_{2} \ln p_{m}} \leq \frac{\delta}{2}\right)$, to prove that $p_{n} \leq O\left(\frac{N}{\ln \ln N}\right)$.

Corollary 4.8. For any $n \in \mathbb{N}$ there exists $N(n)$ such that for any $N>$ $N(n)$ there exists an n-stapling covering.

Proof. Take $\delta=\frac{1}{n}$. Then the result follows from Theorem 4.7.

Theorem 4.7 provides a basis for a stronger and more general result obtained in [8].

\section{Open Problems}

The concepts of stapled sequences and stapling coverings introduced and discussed above lead to some unsolved problems, as follows.

1. What is the exact relationship of cutoff and expense? Can we find a function $f(\varepsilon)=\min _{\varepsilon(T)=\varepsilon} u(T)$ and an algorithm that allows us to transform a stapling covering of a given expense into a stapling covering with the cutoff $u(T)=f(\varepsilon)$ ?

2. Do there exist constructions for efficient stapling coverings of any cutoff $u(T)>0$ that start working for reasonable values of $N$ ? For example, the construction obtained using Erdös' result, even for $u(T)=0.5$, starts working only for values of $N>10^{1000}$. The algorithm in this paper provides such construction for the values of $u(T)=\frac{7}{32}+\varepsilon$ that starts working for $N$ of order of $10^{4}$, but its generalization for any $u(T)>0$ seems to be cumbersome.

\section{Acknowledgments}

The author would like to thank Prof. Paul Erdös and Prof. Ricard K. Guy for providing important references to relevant work. She is also grateful to Prof. Peter Gacs for his invariable support and advice, to Dr. David Bernstein for interest and encouragement, to Prof. Heiko Harborth for his interest in 
THE ELECTRONiC JOURNAL of COMBinAtorics 3 (1996), \#R33

n-stapling coverings, and to Prof. Lev B. Levitin for useful discussions and comments. Special thanks are due to the referee for carefully reading the paper and suggesting thoughtful improvements and to Prof. Herbert S. Wilf for valuable advice.

\section{References}

[1] Alfred Brauer On a Property of $\mathrm{k}$ Consecutive Integers. Bull. Amer. Math. Society, vol. 47, 1941, pp. 328-331.

[2] Ronald J. Evans On Blocks of N Consecutive Integers. Amer. Math. Monthly, vol. 76, 1969, pp.48-49.

[3] Ronald J. Evans On N Consecutive Integers in an Arithmetic Progression. Acta Sci. Math. Univ. Szeged, vol. 33, 1972, pp. 295-296.

[4] Paul Erdös, personal communication.

[5] Paul Erdös, Some remarks on Number Theory. Israel Journal of Mathematics, vol.3, 1965, pp. 6-12.

[6] Paul Erdös, On the Difference of Consecutive Primes. Quarterly Journal of Mathematics, vol.6, 1935, pp. 124-128.

[7] Paul Erdös, personal communication.

[8] Irene Gassko Stapling Coverings of Natural Numbers Without Small Primes. Preprint.

[9] Ricard K. Guy Unsolved Problems in Number Theory. SpringerVerlag, New-York - Heidelberg - Berlin, 1981.

[10] Heiko Harborth Eine Eigenschaft Aufeinanderfolgender Zahlen. Arch. Math. (Basel), vol. 21, 1970, pp.50-51.

[11] Heiko Harborth Sequenzen Ganzer Zahlen. Zahlentheorie, Berichte aus dem Math. Forschungsinst. Oberwolfach, vol. 5, 1971, pp. 59-66.

[12] A.E. Ingham The Distibution of Prime Numbers. Cambridge University Press, Cambridge, 1990. 
THE ELECTRONiC JOURNAL of COMBinAtorics 3 (1996), \#R33

[13] S.S Pillai On m Consecutive Integers I, Proc. Indian Acad. Sci., Sect. A, vol. 11, 1940, pp.6-12; MR 1, 199; II ibid., vol. 11, 1940, pp.73-80, III ibid, vol.13, 1941, pp.530-533; MR 3, 66; IV Bull. Calcutta Math. Soc. 36, 1944, pp. 99-101; MR 6, 170.

\section{Appendix}

\section{A Proofs of Lemmata 3.1, 3.2, 3.3.}

Lemma 3.1. If $S_{N}^{0}$ is a stapled $S S N$ and $T\left(S_{N}^{0}, W_{I}^{0}\right)$ is the corresponding stapling covering, there exists stapling covering $T\left([1, N], W_{I}\right)$ such that $s_{h_{i}}=$ $h_{i}=h_{i}^{0}$ for any $i \in I, s_{h_{i}} \in[1, N]$.

Proof. Let $S_{N}^{0}=\left(s_{1}^{0}, s_{2}^{0}, \ldots, s_{N}^{0}\right), h_{i}^{0}$ be the first indicator of $A_{p_{i}}^{0}$ in $S_{N}^{0}$. Since $T\left(S_{N}^{0}, W_{I}^{0}\right)$ is a stapling covering, $s_{h_{i}^{0}+p_{i}}^{0} \in S_{N}^{0}$, hence $h_{i}^{0}+p_{i} \leq N$. Consider now $W_{I}=\left\{A_{p_{i}}\right\}$, where $A_{p_{i}}=\left\{h_{i}^{0}+m p_{i}\right\}, m \in \mathbb{Z}$. Obviously $h_{i}^{0} \in A_{p_{i}}, h_{i}^{0}+p_{i} \in A_{p_{i}}$ and $h_{i}^{0}+p_{i} \leq N$ for any $i \in I$. Moreover, since for any $k \in[1, N]$ there exists $A_{p_{i}}^{0}$ such that $s_{k}^{0}=s_{r_{i}}^{0} \in A_{p_{i}}^{0}$, it follows that $k=$ $r_{i} \in A_{p_{i}}$. Thus $T\left([1, N], W_{I}\right)$ is a stapling covering and $s_{h_{i}}=h_{i}=h_{i}^{0}, i \in I$ are the first indicators.

Lemma 3.2. If $T\left(S_{N}, W_{I}\right)$ is a stapling covering, then there exists a stapled $S S N S_{N}^{0}$ of length $N$.

Proof. Let $A_{p_{i}}=\left\{a_{p_{i}}+m p_{i}\right\}$, where $a_{p_{i}} \in \mathbb{Z}_{p_{i}}, m \in \mathbb{Z}, i \in I$. Take number $M \in \mathbb{N}$, such that $M \equiv-a_{p_{i}}\left(\bmod p_{i}\right)$ for any $i \in I$. Such a number exists according to the Chinese Remainder Theorem. Let $s_{k}^{0}=s_{k}+M \quad(k \in[1, N])$ and define $S_{N}^{0}$ as $S_{N}^{0}=\left(s_{1}^{0}, s_{2}^{0}, \ldots, s_{N}^{0}\right)$. Obviously, if $s_{k} \in A_{p_{i}}$, then $s_{k}^{0} \in A_{p_{i}}^{0}$ for any $k \in[1, N]$ and for any $i \in I$. Hence, $T\left(S_{N}^{0}, W_{I}^{0}\right)$ is a stapling covering and $h_{i}=h_{i}^{0}$ for any $i \in I$. Thus, $S_{N}^{0}$ is a stapled SSN of length $N$.

Lemma 3.3. $S_{N} \sim S_{N}^{\prime}\left(\operatorname{resp} W_{I}\right)$ iff $s_{k}^{\prime} \equiv s_{k}\left(\bmod \prod_{i \in I} p_{i}\right), \forall k \in$ $[1, N]=(1,2, \ldots, N), s_{k} \in S_{N}, s_{k}^{\prime} \in S_{N}^{\prime}$.

Proof. If $s_{k}^{\prime} \equiv s_{k}\left(\bmod \prod_{i \in I} p_{i}\right)$, then, obviously, $s_{k} \in A_{p_{i}}$ iff $s_{k}^{\prime} \in A_{p_{i}}$.

Thus, $h_{i}=h_{i}^{\prime}$ for any $i \in I$. Conversely, if $h_{i}=h_{i}^{\prime}$ for any $i \in I$, then $s_{h_{i}} \equiv s_{h_{i}}^{\prime}\left(\bmod p_{i}\right)$ for any $i \in I$. Since $s_{k}-s_{h_{i}}=s_{k}^{\prime}-s_{h_{i}}^{\prime}=k-h_{i}$ for any 
THE ELECTRONiC JOURNAL of COMBinAtorics 3 (1996), \#R33

$k \in[1, N]$, it follows that $s_{k}^{\prime} \equiv s_{k}\left(\bmod p_{i}\right)$ for any $i \in I$, and, therefore, $s_{k}^{\prime} \equiv s_{k}\left(\bmod \prod_{i \in I} p_{i}\right)$.

\section{B Proof of Lemma 4.1.}

Lemma 4.1. Consider the set $Q=\left\{2^{s} 3^{t} \mid s, t \in \mathbb{N}, 2^{s} 3^{t} \leq N\right\}$.

Then $|Q| \leq \frac{1}{2} \log _{2} N\left(\log _{3} N-1\right)$ for any $N \geq 9$.

Proof Obviously,

$$
|Q|=\sum_{k=1}^{v}\left\lfloor\log _{2} \frac{N}{3^{k}}\right\rfloor
$$

where $v=\left\lfloor\log _{3} N\right\rfloor$

Let

$$
\log _{2} \frac{N}{3^{k}}-\left\lfloor\log _{2} \frac{N}{3^{k}}\right\rfloor=\varepsilon
$$

and

$$
\log _{2} \frac{N}{3^{k+1}}-\left\lfloor\log _{2} \frac{N}{3^{k+1}}\right\rfloor=\delta,
$$

where $0 \leq \varepsilon<1,0 \leq \delta<1$.

Suppose $2^{m} \leq \frac{N}{3^{k}}<2^{m+1}$.

Then

$$
\log _{2} \frac{N}{3^{k} 2^{m}}=\varepsilon
$$

and

$$
\delta=\log _{2} \frac{N}{3^{k+1} 2^{m-h}}=\varepsilon-\log _{2} 3+h,
$$

where $\mathrm{h}$ is an integer such that $0 \leq \delta<1$. Hence, if $\varepsilon \in\left[0, \log _{2} 3-1\right)$, then $h=2$; if $\varepsilon \in\left[\log _{2} 3-1,1\right), h=1$. Thus

$$
\delta= \begin{cases}2+\varepsilon-\log _{2} 3, & 0 \leq \varepsilon<\log _{2} 3-1 \\ 1+\varepsilon-\log _{2} 3, & \log _{2} 3-1 \leq \varepsilon<1\end{cases}
$$


It is easy to infer from (B.4) that $\min _{0 \leq \varepsilon<1}(\varepsilon+\delta)=2-\log _{2} 3$.

Therefore,

$$
\sum_{k=1}^{v}\left(\log _{2} \frac{N}{3^{k}}-\left\lfloor\log _{2} \frac{N}{3^{k}}\right\rfloor\right) \geq\left\lfloor\frac{v}{2}\right\rfloor\left(2-\log _{2} 3\right)
$$

On the other hand,

$$
\sum_{k=1}^{v} \log _{2} \frac{N}{3^{k}}=v\left(\log _{2} N-\frac{v+1}{2} \log _{2} 3\right)
$$

Denote: $\log _{3} N-v=\alpha$. Then

$$
\sum_{k=1}^{v} \log _{2} \frac{N}{3^{k}}=\frac{1}{2} \log _{2} N \log _{3} N-\frac{1}{2} \log _{2} N+\left(\frac{\alpha}{2}-\frac{\alpha^{2}}{2}\right) \log _{2} 3
$$

But $\frac{1}{2} \log _{2} 3 \alpha(1-\alpha) \leq \frac{1}{8} \log _{2} 3$. Hence,

$$
\sum_{k=1}^{v} \log _{2} \frac{N}{3^{k}} \leq \frac{1}{2} \log _{2} N\left(\log _{3} N-1\right)+\frac{1}{8} \log _{2} 3
$$

From (B.5) and (B.7), we obtain:

$$
\sum_{k=1}^{v}\left\lfloor\log _{2} \frac{N}{3^{k}}\right\rfloor \leq \frac{1}{2} \log _{2} N\left(\log _{3} N-1\right)-\left\lfloor\frac{v}{2}\right\rfloor\left(2-\log _{2} 3\right)+\frac{1}{8} \log _{2} 3
$$

Finally, for $v \geq 2$, i.e. for $N \geq 9$, it follows that

$$
|Q| \leq \frac{\log _{2} N\left(\log _{3} N-1\right)}{2}
$$

which proves Lemma 4.1 .

Further analysis of expressions (B.5) and (B.6) allows us to obtain a stronger inequality:

$$
|Q| \leq \begin{cases}\frac{1}{2}\left[\log _{2} N\left(\log _{3} N-2\right)+\log _{3} 2-\log _{2} 3\right] & \text { if }\left\lfloor\log _{3} N\right\rfloor \text { is even } \\ \frac{1}{2}\left[\log _{2} N\left(\log _{3} N-2\right)+\log _{3} 2-\log _{2} 3\right]+1 & \text { if }\left\lfloor\log _{3} N\right\rfloor \text { is odd }\end{cases}
$$


THE ELECTRONiC JOURNAL of COMBinAtorics 3 (1996), \#R33

\section{Proof of Lemma 4.4.}

Lemma 4.4 Let

$G=\left\{x\left|x= \pm 2^{s} 3^{t} 5^{v},\right| x \mid \leq \frac{N}{2} ; s \in \mathbb{N} ; t, v \in \mathbb{N} \cup 0 ; x \not \equiv 1(\bmod 3)\right\}$

Then

$$
|G|<\frac{1}{3} \log _{2} \frac{N}{2} \log _{3} \frac{N \sqrt{5}}{2} \log _{5} \frac{5 N}{2}+1
$$

Proof Denote $Z(n)=\left\{ \pm 2^{s} 3^{t} \mid 2^{s} 3^{t} \leq n ; n, s, t \in \mathbb{N}\right\} \cup Y(n)$, where $Y(n)=\left\{y\left|y= \pm 2^{s},\right| y \mid \leq n, s \in \mathbb{N}, y \equiv 2(\bmod 3)\right\}$.

Since exactly one of two numbers, $2^{s}$ or $-2^{s}$, belongs to $Y(n),|Y(n)|=\left\lfloor\log _{2} n\right\rfloor$. By Lemma 4.1,

$$
|Z(n)| \leq \log _{2} n\left(\log _{3} n-1\right)+\left\lfloor\log _{2} n\right\rfloor \leq \log _{2} n \log _{3} n
$$

By Lemma 4.1, (C.3) is valid for $n \geq 9$. However, direct checking shows that (C.3) is valid for all $n \geq 3$. For $n=2,|Z(2)|-\log _{2} 2 \log _{3} 2=1-\log _{3} 2$.

Obviously,

$$
|G|=\sum_{k=0}^{w}\left|Z\left(\left\lfloor\frac{N}{2 \cdot 5^{k}}\right\rfloor\right)\right| \leq \sum_{k=0}^{w} \log _{2} \frac{N}{2 \cdot 5^{k}} \log _{3} \frac{N}{2 \cdot 5^{k}}+1-\log _{3} 2
$$

where $w=\left\lfloor\log _{5} \frac{N}{2}\right\rfloor$.

Consider the integral

$$
\int_{0}^{\log _{5} \frac{N}{2}} \log _{2} 5 \log _{3} 5\left(\log _{5} \frac{N}{2}-x\right)^{2} d x=\frac{1}{3} \log _{2} \frac{N}{2} \log _{3} \frac{N}{2} \log _{5} \frac{N}{2}
$$

On the other hand,

$$
\begin{gathered}
\int_{0}^{\log _{5} \frac{N}{2}} \log _{2} 5 \log _{3} 5\left(\log _{5} \frac{N}{2}-x\right)^{2} d x=\sum_{k=0}^{w} \int_{k-1}^{k} \frac{N}{2} \log _{2} 5 \log _{3} 5\left(\log _{5} \frac{N}{2}-x\right)^{2} d x+ \\
\int_{w}^{\log _{5} \frac{N}{2}} \log _{2} 5 \log _{3} 5\left(\log _{5} \frac{N}{2}-x\right)^{2} d x=\sum_{k=0}^{w}\left[\log _{2} \frac{N}{2 \cdot 5^{k}} \log _{3} \frac{N}{2 \cdot 5^{k}}+\log _{2} 5 \log _{3} \frac{N}{2 \cdot 5^{k}}\right. \\
+\frac{1}{3} \log _{2} 5 \log _{3} 5+\frac{1}{3} \log _{2} 5 \log _{3} 5\left(\log _{5} \frac{N}{2}-w\right)^{3}
\end{gathered}
$$


It follows from (C.3),(C.4), and (C.6), that

$$
\begin{aligned}
|G| \leq & \frac{1}{3} \log _{2} \frac{N}{2} \log _{3} \frac{N}{2} \log _{5} \frac{N}{2}+\log _{2} \frac{N}{2} \log _{3} \frac{N}{2}-w\left(\log _{3} \frac{N}{2}-\frac{w+1}{2} \log _{3} 5\right) \log _{2} 5 \\
& -\frac{w}{3} \log _{2} 5 \log _{3} 5-\frac{1}{3} \log _{2} 5 \log _{3} 5\left(\log _{5} \frac{N}{2}-w\right)^{3}+1-\log _{3} 2
\end{aligned}
$$

Denote $\log _{5} \frac{N}{2}-w=\beta ; 0 \leq \beta<1$. Then

$$
\begin{aligned}
|G| \leq & \frac{1}{3} \log _{2} \frac{N}{2} \log _{3} \frac{N}{2} \log _{5} \frac{N}{2}+\frac{1}{2} \log _{2} \frac{N}{2} \log _{3} \frac{N}{2}+\frac{1}{6} \log _{2} \frac{N}{2} \log _{3} 5- \\
& \log _{2} 5 \log _{3} 5\left(\frac{1}{6} \beta-\frac{1}{2} \beta^{2}+\frac{1}{3} \beta^{3}\right)+1-\log _{3} 2
\end{aligned}
$$

It is easy to show that

$$
\min _{0 \leq \beta<1}\left(\frac{1}{6} \beta-\frac{1}{2} \beta^{2}+\frac{1}{3} \beta^{3}\right)=-\frac{1}{36 \sqrt{3}}
$$

Thus

$$
\begin{aligned}
|G| & \leq \frac{1}{3} \log _{2} \frac{N}{2} \log _{3} \frac{N \sqrt{5}}{2} \log _{5} \frac{5 N}{2}+\frac{\log _{2} 5 \log _{3} 5}{36 \sqrt{3}}+1-\log _{3} 2 \\
& <\frac{1}{3} \log _{2} \frac{N}{2} \log _{3} \frac{N \sqrt{5}}{2} \log _{5} \frac{5 N}{2}+1
\end{aligned}
$$

which proves Lemma 4.4 . 